\title{
What Do Older Adults and Clinicians Think About Traditional Mobility Aids and Exoskeleton Technology?
}

\author{
MEREL M. JUNG, Jheronimus Academy of Data Science and Tilburg University, The Netherlands \\ GEKE D. S. LUDDEN, University of Twente, The Netherlands
}

\begin{abstract}
Mobility impairments can prevent older adults from performing their daily activities, which highly impacts quality of life. Powered exoskeletons, which are wearable robotic devices, can assist older adults by providing additional support to compensate for age-related decline in muscle strength. To date, little is known about the opinions and needs of older adults regarding exoskeletons, as current research primarily focuses on the technical development of exoskeleton devices and on groups with more severe mobility impairments such as people with spinal cord injuries. Therefore, the aim of this article is to inform the design of exoskeletons for older adults from a person-centered perspective. Interviews were conducted with seven older adults and six clinicians. Results indicated that exoskeletons can be a valuable addition to existing mobility devices to support older adults during walking and other actions necessary to perform their daily activities. Although reactions towards the concept of exoskeleton technology were positive, older adults did not currently perceive the need for an exoskeleton device. Exoskeletons for older adults should be easy to use; preferably, users should be able to put the device on and take it off independently. Moreover, the appearance of the exoskeleton should be as inconspicuous as possible, as most older adults do not like to advertise their need for assistive devices. At this point in time, the willingness to use exoskeleton technology will depend on personal needs and preferences.
\end{abstract}

CCS Concepts: • Social and professional topics $\rightarrow$ Assistive technologies; Seniors; $\bullet$ Human-centered computing $\rightarrow$ User centered design;

Additional Key Words and Phrases: Older adults, assistive technology, exoskeleton, user perspective, mobility ACM Reference format:

Merel M. Jung and Geke D. S. Ludden. 2019. What Do Older Adults and Clinicians Think About Traditional Mobility Aids and Exoskeleton Technology? ACM Trans. Hum.-Robot Interact. 8, 2, Article 10 (May 2019), 17 pages.

https://doi.org/10.1145/3311789

\section{INTRODUCTION}

In an aging population, mobility impairments are prevalent due to a decline in physical functioning and muscle strength $[33,37,40]$. When physical deficiencies make it increasingly difficult to move, people stop moving, which increases both the risk of diseases associated with a sedentary lifestyle (e.g., cardiovascular disease, type 2 diabetes, and obesity) and the risk of withdrawing

Authors' addresses: M. M. Jung, Jheronimus Academy of Data Science and Tilburg University, Cognitive Science \& Artificial Intelligence, Warandelaan 2, 5037 AB, Tilburg, The Netherlands; email: m.m.jung@uvt.nl; G. D. S. Ludden, University of Twente, Chair of Interaction Design, P.O. Box 217, 7500 AE, Enschede, The Netherlands; email: g.d.s.ludden@utwente.nl. Permission to make digital or hard copies of all or part of this work for personal or classroom use is granted without fee provided that copies are not made or distributed for profit or commercial advantage and that copies bear this notice and the full citation on the first page. Copyrights for components of this work owned by others than the author(s) must be honored. Abstracting with credit is permitted. To copy otherwise, or republish, to post on servers or to redistribute to lists, requires prior specific permission and/or a fee. Request permissions from permissions@acm.org.

(C) 2019 Copyright held by the owner/author(s). Publication rights licensed to ACM.

2573-9522/2019/05-ART10

https://doi.org/10.1145/3311789 
from participation in society [33, 37, 40]. Moreover, mobility loss prevents people from performing their daily activities, which highly impacts the quality of life of a person and often increases the dependency on others [22, 37, 40]. In light of the aforementioned concerns, the World Health Organization has proposed a framework for action towards active aging, which is defined as "the process of optimizing opportunities for health, participation and security to enhance quality of life as people age" [39].

To stay active while aging, assistive technology can help individuals with reduced mobility to perform their daily activities safely and independently [7, 15, 34]. Importantly, unnecessary wheelchair use should be avoided to prevent loss of muscle strength as a result of inactivity [15]. Increasing the mobility of older adults ${ }^{1}$ can increase their level of physical activity and can have a positive effect on their well-being and cognitive functioning [28, 33, 40]. However, traditional mobility aids have their disadvantages. For example, the rollator, commonly used by older adults, has been criticized for being heavy and bulky, making it difficult to handle, park, and transport the device $[5,34]$. Additionally, most walking aids such as canes and walkers do not provide sufficient support during sit-to-stand transfers, which are an integral part of daily life [29]. For these reasons, powered exoskeletons, which are wearable robotic devices, might be a suitable mobility aid for older adults.

The aim of the study presented in this article is to inform the design of exoskeleton devices for older adults. Currently, exoskeletons are being developed for the military, industry, and for medical applications such as rehabilitation [8]. As technology advances, exoskeletons can also support older adults with daily living by providing additional support during standing, walking, sit-tostand transfers, and climbing stairs to compensate for age-related decline in muscle strength [1, 29]. Besides the technical developments of exoskeletons, it is important to focus on the perspective of the user [9]. User acceptance and usability are critical factors in the successful adoption of any assistive technology, therefore it is important to involve end-users and other stakeholders in the development process $[5,7,9,24,34,38]$. To this end, we will explore the views of older adults and clinicians on exoskeleton technology to assist with daily living.

\section{RELATED WORK}

\subsection{Advances in the Development of Exoskeleton Technology}

Several exoskeleton prototypes are being developed to assist people with mobility impairments including older adults. Age-related changes in gait function (e.g., decreased walking speed, shorter step length, increased step variability, and reduced range of motion at the hip, knee, and ankle joints) and decreased metabolic efficiency for walking strongly affect the ability to perform daily activities [13, 31]. Improving walking function is therefore an important focus when developing exoskeletons for older adults. Lab studies with older adults showed that the Gait Enhancing Mechatronic System (GEMS) - a wearable hip exoskeleton that provides walking assistance-can improve gait function and cardiopulmonary metabolic efficiency and reduce muscle effort $[13,14]$. In other work, seven older adult men walked on a treadmill at a constant speed for 50 minutes both with and without wearing a robotic hip exoskeleton called the Stride Assistance System (SAS) [31]. Results showed that the SAS significantly improved walk ratios (i.e., step length/steps per minute), walking speeds, and step lengths without increasing energy consumption of the leg muscles. In a long-term study, 15 older women participated in a three-month supervised walking program using the SAS [30]. The results showed that a walking intervention with the SAS can improve walking speed and muscle efficiency.

\footnotetext{
${ }^{1}$ We will use the term "older adults" to refer to people aged $65+$.
} 
Researchers have also tried to integrate exoskeleton technology with existing mobility aids to come up with innovative solutions. For example, the EXPOS is a lightweight exoskeleton designed to support older adults and rehabilitating patients to sit down, stand up, and walk [12]. The exoskeleton is attached to a walker that carries the electronics, thereby reducing the weight of the exoskeleton. Another concept for a hybrid mobility device is the COMBO, which offers wheelchair users the health benefits of standing and walking through an attached powered exoskeleton while also maintaining the advantages of wheeled mobility [4].

More recently, soft exoskeletons-often referred to as exosuits-are being developed by replacing rigid structures with garment-like textiles integrated with compliant sensors and actuators to interface with the human body in a more comfortable manner $[2,36]$. These devices can be made significantly lighter and more portable [29,36]. Jin et al. showed that older adults can benefit from wearing a soft robotic suit that provides assistive force for hip flexion during walking, both in terms of reduced energy expenditure and improved gait characteristics [11]. Another example is the Myosuit, which is designed to provide anti-gravity support during standing, walking, and sit-to-stand transfers to assist people with mobility impairments during their daily activities [29].

Users of exoskeletons will be interacting closely with the device, therefore the control interface should be intuitive, seamless, and non-obtrusive [7]. One way of adapting to the needs of people is by providing the appropriate level of assistance based on the capabilities of a person and the performed action [1]. As a result of this method, the exoskeleton only compensates for the muscle weakness of the user, which stimulates the use of remaining muscle strength, preventing unnecessary disuse. The aforementioned studies show promising results regarding the benefits of exoskeletons in terms of improving gait characteristics and reducing energy expenditure. However, older adults will only be able to benefit from exoskeleton technology if they are willing and able to use such a device [9].

\subsection{User Perspective on the Use of Exoskeleton Technology}

Some studies have focused on the user perspective regarding the use of exoskeletons for target groups with more severe mobility impairments such as people with spinal cord injuries and those recovering from a stroke. For example, Wolff et al. conducted a survey on the use of exoskeletons among wheelchair users and care professionals [38]. Results showed that the most important reason for wheelchair users to use an exoskeleton would be for the health benefits associated with standing and walking. Notably, also psychosocial benefits from the use of exoskeletons were mentioned; for example, the opportunity to engage in eye-level social interaction. Stakeholders placed high importance on safety, purchase and maintenance costs, comfort, and the ease of use putting on/taking off the exoskeleton. The appearance of an exoskeleton device and required training time were deemed less important. Currently, it is unclear whether these findings can be generalized to other target groups such as older adults. Differences in age (i.e., $88 \%$ of the wheelchair users in the study were under the age of 65) and health condition between the two user groups might result in different opinions and needs regarding the use of exoskeleton technology.

To date, little is known about the opinions and needs of older adults with respect to exoskeleton technology for home use, as this topic has only been addressed by a few studies. For example, in a questionnaire, older adults rated the importance of different body motions needed to perform their daily activities [22]. The results indicated that assistance would be required during actions such as sitting down, standing up, standing, lifting, carrying, and bending. In other work, the European XoSoft project aims to develop an exosuit for the lower body to assist (older) adults with mobility impairments [25]. Potential users (i.e., people recovering from a stroke, people with incomplete spinal cord injuries, and older adults) and their (in)formal caregivers were asked to give their opinions on the XoSoft concept design. The soft wearable assistive device was generally well 
received, although frail older adults tended to be less positive about the concept. The main design requirements listed were comfort (e.g., lightweight, compatible with usual clothing/footwear, and the option to wear the device underneath normal clothing), ease of use (e.g., independent donning and doffing, easy to clean, not bulky, and no extensive training required), and affordability (either by a low purchasing cost or reimbursement).

In contrast to previous studies, we will specifically focus on the needs and preferences of older adults, and we will explore the views on exoskeleton technology in general instead of asking for opinions on one specific exoskeleton concept. The aim of the study presented in this article is to inform the design of exoskeleton technology that can help older adults with reduced mobility to perform their daily activities. In addition, we will explore the most promising situations in which older adults would use such a device.

\section{METHODS}

\subsection{Participants}

Participants were recruited through a contact person at an assisted-living facility in the eastern part of the Netherlands who gathered a list of suitable candidates among staff and residents. In addition, participants were recruited via a mobile application that was developed by a local organization supporting the well-being of older adults. Exclusion criteria were the presence of any cognitive or sensory impairments that would limit the ability to participate in the study. Interviews were conducted with seven older adults (two male, five female) and six clinicians (two male, four female) who all volunteered to take part. The age of the older adults ranged between 68 and $94(M=85, S D=9)$. Most older adults (six) resided in an assisted-living facility and received help with activities such as dressing, cleaning, and grocery shopping, whereas the one other participant lived independently. None of the older adults were users of exoskeleton technology. The age of the clinicians ranged between 27 and $68(M=39, S D=15)$. Their experience with older adults working as a physical therapist (three), occupational therapist (one), or nurse (two) ranged from 2 to 44 years $(M=14, S D=16)$. Written informed consent was obtained from all participants. The ratings of their general interest in technology on a scale ranging from 1 (not interested) to 5 (very interested) were lower for the older adult participants $(\operatorname{Mdn}(Q 1, Q 3)=3(2.5,4))$ compared to the clinicians $(\operatorname{Mdn}(Q 1, Q 3)=4(3.25,4))$. All older adults and most clinicians (four/six) were unfamiliar with the concept of exoskeletons (all rated 1-I have never heard of exoskeleton technology). The other two clinicians reported to have seen exoskeletons in pictures and/or video (one participant rated 2-I am somewhat familiar with the concept, and the other rated 3-I am very familiar with the concept).

\subsection{Materials}

3.2.1 Interview. A semi-structured one-on-one interview was conducted in two versions to either cover the personal experiences of older adults or the experiences of clinicians who are working with older adults. During the interview, the researcher took notes and audio recordings were made for later analysis. The interview consisted of three parts, and all interviews were conducted in Dutch. Part one covered basic demographic information: age, gender, profession, years of experience working in elderly care (clinicians only), and living situation (older adults only). Also, general interest in technology was rated on a 5-point Likert scale ranging from 1-not interested to 5-very interested.

Part two covered questions on mobility. Older adults were asked the following questions: "Do you experience mobility difficulties in daily life? If so, what kind of difficulties?" and "Do you use walking aids? If so, what kind?." To explore their opinions on traditional mobility aids, the following questions were asked about each of the devices that they used: (a) "In which situations/with 
what goal do you use this walking aid?" (b) "What are in your experience the advantages of this walking aid?" (c) "What are in your experience the disadvantages of this walking aid?." Clinicians were asked the following questions: "What are the most prominent mobility difficulties that are experienced by your clients in their daily lives?" and "Which walking aids are most often used by your clients?" For each of the three most used walking aids, the following questions were asked: (a) "In which situations/with what goal do your clients use this walking aid?" (b) "What are in your experience the advantages of this walking aid?" (c) "What are in your experience the disadvantages of this walking aid?.”

Part 3 covered questions on exoskeleton technology, including an introduction to the technology (see Section 3.2.3). Both the older adults and the clinicians were asked the following questions: "How familiar are you with exoskeleton technology?" Similar to the question included in the survey by Reference [38], participants were asked to rate their familiarity on a 4-point Likert scale (1-I have never heard of exoskeleton technology; 2-I am somewhat familiar with the concept; $3-\mathrm{I}$ am very familiar with the concept; 4-I would consider myself an expert on exoskeleton technology). The next questions regarded the positive and negative aspects of exoskeleton technologies: "What are in your opinion the positive aspects of exoskeletons?" and "What are in your opinion the negative aspects of exoskeletons?." In addition, older adults were asked the following questions: "Would you be interested in using an exoskeleton in your daily life? If so, in which situations/with what goal would you use such a device? If not, why?" and "Do you know other older adults that might be interested in using an exoskeleton in their daily lives? If so, in which situations/with what goal would they use such a device? If not, why?." Clinicians were asked the following questions: "Would your clients be interested in using an exoskeleton in their daily lives? If so, in which situations/with what goal would they use such a device? If not, why?."

3.2.2 Questionnaire. The level of mobility of the older adults was assessed through a questionnaire. The questionnaire was adapted from a Dutch questionnaire assessing the capacities of patients with rheumatoid arthritis to perform their daily activities [3]. Only the subscales related to mobility were included covering questions about walking (i.e., walking outside for 15 minutes and stair climbing) and activities (i.e., grocery shopping, getting in and out of a car, and performing household chores). Participants were asked to rate their ability to perform these actions or activities in the last week on a 4-point Likert scale (0-independently without difficulty; 1independently with some difficulty; 2-independently with great difficulty; 3-unable to perform independently). In addition, participants were asked to indicate which mobility aids they had used performing these actions or activities.

3.2.3 Introduction to Exoskeleton Technology. Participants were introduced to the concept of exoskeletons by a short explanation, including pictures (see Figure 1) and a short video featuring people wearing different exoskeleton devices. The introduction to the technology covered the development of exoskeleton devices over the past decades illustrating the progress in design by showing a picture of the Hardiman ${ }^{2}$ by General Electric next to a modern exoskeleton. In addition, the following topics were covered: application areas (i.e., military, industry, and medical), examples of actions that can be supported (i.e., sit-to-stand transfers, bending, walking), and research into soft exoskeletons. Pictures of existing mobility aids (i.e., cane, walker, rollator) were also shown accompanied by the question of whether exoskeletons could be a suitable addition. The aim of this question was to elicit participants to think about the possibility. To give a better idea of the workings of exoskeletons, a 1.5-minute video clip was shown on a laptop computer covering a woman walking wearing a hip exoskeleton (Stride Management Assist by Honda; see Figure 1, right) and

\footnotetext{
${ }^{2}$ https://www.ge.com/reports/post/78574114995/the-story-behind-the-real-iron-man-suit/.
} 

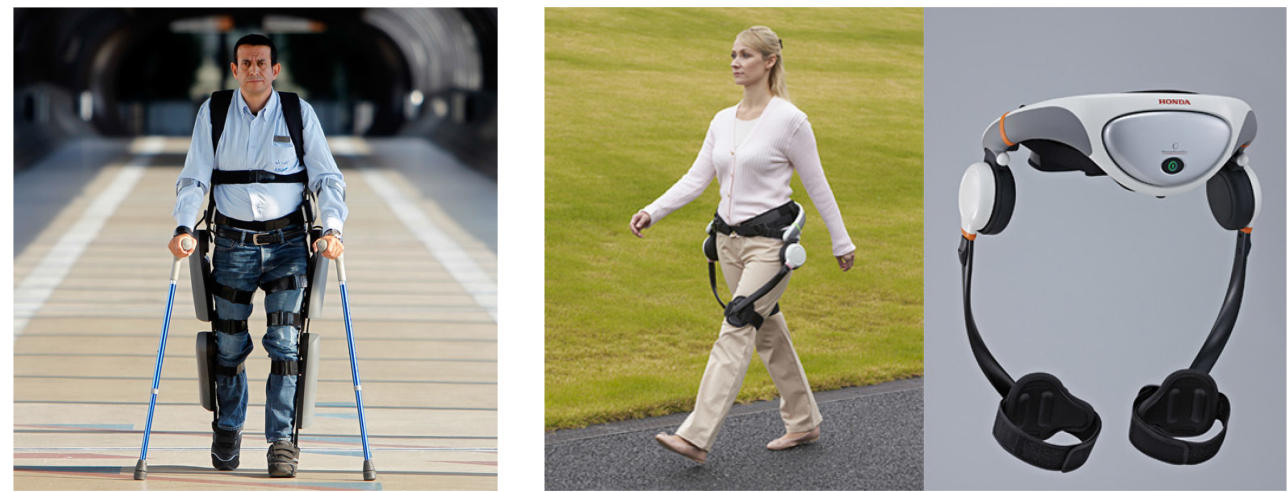

Fig. 1. Examples of exoskeletons that were shown to participants to introduce the technology (left to right): ReWalk $^{\mathrm{TM}}$ exoskeleton [27], Stride Management Assist by Honda [10].

a man performing several actions wearing a full lower-body exoskeleton (Keeogo ${ }^{\text {TM }}$ by B-TEMIA). The video was compiled from two corporate videos available online. ${ }^{3}$ Sound was removed to avoid possible language barriers, as both videos were in English.

\subsection{Procedure}

Participants were individually welcomed by the researcher in a quiet office within either the care facility where the participant worked/lived or at the University of Twente. The participants were informed about the nature of the study and were given the opportunity to ask questions before signing the informed consent form. Next, audio recordings were started and part one of the interview was conducted followed by the questionnaire (older adults only). Then, part two of the interview was conducted, followed by the first question of part three regarding familiarity with exoskeleton technology. Subsequently, participants were introduced to the concept of exoskeletons followed by the remaining questions of part three of the interview. Afterwards, participants were given the opportunity to provide any additional comments and ask questions. The entire procedure took approximately 30 minutes.

\subsection{Data Analysis}

Notes and audio recordings from the interviews were summarized per question using ATLAS.ti ${ }^{4}$ version 8.1.28. Themes were identified from the data using a general inductive approach [35]. The number of participants that mentioned each of the themes was tallied per user group. In the results section, the themes are presented in italics between square brackets and are supported by quotes from the participants. Descriptive statistics of the demographic information and the ratings from interview questions and the questionnaire were calculated using $\mathrm{R}$ [26]. The ratings of the questionnaire were scored according to the instructions of the original questionnaire [3]; that is, scores were calculated by averaging the highest rated item from each of the two subscales (higher scores indicate more difficulties).

\section{RESULTS}

\subsection{Mobility Difficulties and Use of Assistive Devices by Older Adults}

4.1.1 Mobility Questionnaire. The scores of the questionnaire $(\operatorname{Mdn}(Q 1, Q 3)=3(2.25,3))$ showed that most of the older adults experienced mobility difficulties and used mobility aids to

\footnotetext{
${ }^{3}$ https://www.youtube.com/watch?v=Io3MBpzmVJc and https://www.youtube.com/watch?v=-uQs-FJnC4I.

${ }^{4}$ by Scientific Software Development GmbH, http://atlasti.com.
} 
Table 1. Results of the Mobility Questionnaire

\begin{tabular}{ccl}
\hline Older adult & Mobility score $(0-3)^{a}$ & Mobility aid(s) used \\
\hline 1 & 0 & none \\
2 & 3.0 & cane, rollator, mobility scooter \\
3 & 3.0 & rollator \\
4 & 2.5 & cane, rollator \\
5 & 3.0 & wheelchair \\
6 & 2.0 & rollator \\
7 & 3.0 & does not apply ${ }^{b}$ \\
\hline
\end{tabular}

${ }^{a}$ Higher scores indicate more difficulties; ${ }^{b}$ Unable to perform any of the listed activities.

walk and perform activities (see Table 1). Some of the participants were dependent on assistance from other people.

4.1.2 Mobility Difficulties in Daily Activities. Most older adults (6/7) reported that they experienced difficulties performing daily activities due to reduced mobility. Walking, especially longer distances, was difficult for most participants (5/7). In addition, the general remark was made that all daily activities had become more difficult (3/7). Also, most participants (5/7) reported to be careful to avoid falling. The clinicians indicated that their clients had difficulty with walking (especially longer distances and walking outside) (6/6), sit-to-stand transfers (5/6), and standing for prolonged periods of time; for example, when showering or cooking (3/6). Also, the risk of falling was highlighted (3/6).

4.1.3 Use of Mobility Aids. Most older adult participants reported using at least one mobility device (6/7): 2 people only used a rollator, 2 used a rollator and cane, 1 used a rollator, cane, and mobility scooter, and the other participant only used a wheelchair (see Figure 2). The clinicians indicated that the rollator was the most-used mobility device by their clients (6/6). Other mobility aids that were frequently used were mobility scooters $(3 / 6)$, walkers $(2 / 6)$, wheelchairs $(2 / 6)$, crutches (2/6), and a cane (1/6).

4.1.4 Rollator. Older adults reported that they used their rollator both within their home $(5 / 5)$ and outside (4/5). Participants valued the support that the device provides (5/5), helping to prevent falling (2/5) and contributing to a feeling of safety (1/5). Also, the ability to sit down on the device was appreciated (1/5). The only downside mentioned was that people were dependent on their rollator, meaning that they had to take the device everywhere, causing some transportation problems (3/5). The clinicians indicated that rollators were often used to prevent falling $(4 / 6)$ and to increase mobility at home (3/6) and/or outside (3/6). Advantages that were mentioned included the increase in stability (5/6), mobility (4/6), and feeling of safety (2/6). Other positive aspects included the ability to transport items (3/6) and to sit down to rest. However, in order for the device to have a positive effect and to avoid accidents, it is important that the rollator is adjusted to the height of the user and is used properly (2/6). Moreover, sufficient space is needed to allow for the use of a rollator at home (2/6). Also, a rollator might be less suitable for some older adults, as the device can roll away; in this case, a walking frame might be a better option (2/6).

4.1.5 Walking Frame. Clinicians indicated that walking frames are used in similar situations as rollators $(2 / 2)$. Walking frames have the advantage of not rolling away $(2 / 2)$ and providing more support compared to rollators $(1 / 2)$. However, the lack of wheels comes with the disadvantage of needing to lift the device to move around, which requires more effort from the user $(2 / 2)$. 

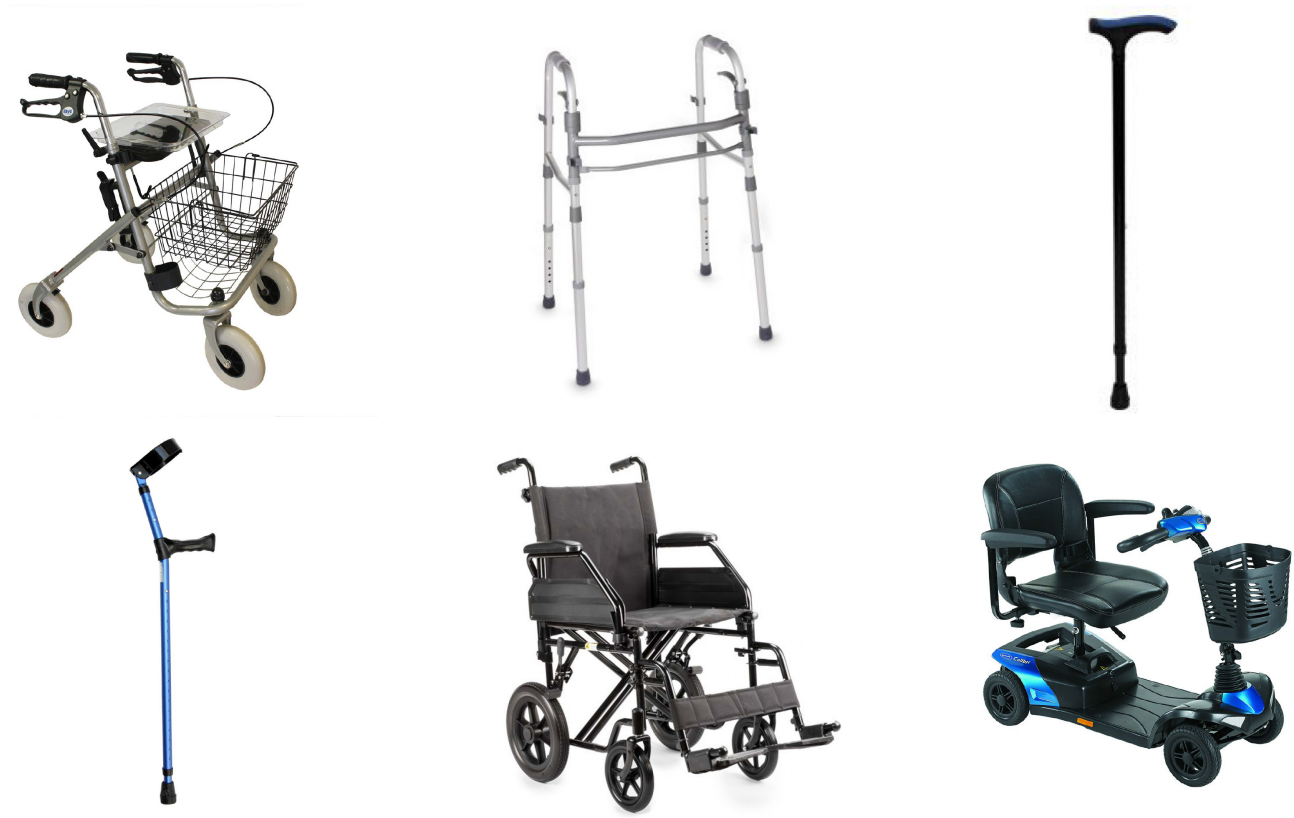

Fig. 2. Commonly used mobility aids (left to right, top to bottom): rollator [19], walking frame [20], cane [16], crutch [17], non-powered wheelchair [21], mobility scooter [18].

4.1.6 Cane. Older adults reported using their cane at home (2/3) and/or outside (2/3). One older adult indicated that it was easier to use a cane when riding the bus instead of taking his rollator with him. The other two participants used their cane predominantly when walking short distances; for example, within their living room. Positive aspects of the cane included the support that the device can provide (2/3) and its compact size (1/3). No disadvantages were reported (3/3), participants indicated that they had gotten used to the device $(2 / 3)$. One of the clinicians indicated that a cane can be used to alleviate some of the weight that needs to be supported by the lower limbs, which can also help to reduce pain (1/1). Moreover, a cane can provide stability, albeit less compared to a rollator or walking frame. The option to use a cane for self-defense was seen as an advantage. Downsides included that the device can easily fall when stored against a wall or table and the risk of the cane sliding away on a slippery surface.

4.1.7 Crutches. Clinicians indicated that one or two crutches can be used to alleviate the pressure put on joints and to increase stability (2/2). One of the participants indicated that older adults are more inclined to accept the use of crutches compared to a rollator or a mobility scooter. Another positive aspect of the device that was mentioned was its compact size (1/2). Disadvantages included the inability of the crutches to stand upright on their own (1/2) and the need of one or both hands to use the device(s) making it harder to open doors (1/2). Moreover, it was mentioned that using crutches to climb stairs can be dangerous $(1 / 2)$ and that the use of crutches is not a suitable long-term solution to mobility problems $(1 / 2)$.

4.1.8 Non-powered Wheelchair. One of the older adults used a wheelchair during the entire day $(1 / 1)$. She mentioned that the wheelchair enabled her to go places, and she saw no disadvantages in using the device. Two of the clinicians indicated that wheelchairs are primarily used to go outside by people that are unable to walk (longer distances) $(2 / 2)$. The main advantage mentioned was the 
social aspect of being able to meet other people and participate in activities (e.g., going shopping together instead of having someone do the shopping for you) $(2 / 2)$. Wheelchairs are seen as a last resort, as maintaining walking function will help to prevent muscle dystrophy $(1 / 2)$. Other disadvantages included the dependency on another person to push the wheelchair, which is a heavy task (1/2). Also, the person in the wheelchair might get cold sitting still for a prolonged period of time $(1 / 2)$.

4.1.9 Mobility Scooter. One of the older adult participants used a mobility scooter for shopping and to drive to the waterside to watch the fishermen (1/1). He mentioned that he enjoys the ability to cover long distances (compared to using a rollator) and the benefits of being able to sit. The only negative aspect of the device according to the participant was that he gets cold from sitting still when he goes outside during winter time. Also, he mentioned buying his own mobility scooter, as he was uncomfortable using the one that was provided to him by the municipality. The clinicians indicated that the mobility scooter was primarily used outside (3/3); for example, to go grocery shopping (2/3). Advantages included the freedom and independence that the device can provide $(3 / 3)$ and the ability to cover long distances (2/3). Disadvantages included concerns about safety due to the relatively high speed of the device $(1 / 3)$ and concerns about older adults with cognitive impairments such as dementia using the device $(1 / 3)$.

\subsection{Views on Exoskeleton Technology for Older Adults}

4.2.1 Suitability of Exoskeletons for Older Adults. [Positive about exoskeleton concept] All participants (13/13) were positive about the general concept of exoskeletons.

Clinician 1: "I'm in favor of this [exoskletons]. I'm in favor of these kinds of innovations."

Older adult 2: "I think that it is a good thing that they [exoskeleton devices] exist." Interviewer: "Why do you think that this is such a good thing?" Older adult 2: "Well, some people really need it ..."

Older adult 6: "Imagine that I wouldn't be able to walk anymore and they would be able to make me walk again with this [exoskeleton]. [... ] Yes, I think it's a positive thing [exoskeleton technology], I really mean it. People would like to live their life. If you would be able to walk with something like this [exoskeleton], people would want to walk."

[Would use exoskeleton if necessary] Most older adults (5/7) indicated that they would wear an exoskeleton device if needed. However, none of these participants felt that they needed such a device at the moment.

Older adult 2: "As long as it [the use of an exoskeleton device] is not necessary, I would not use it." Interviewer: "And if you would need it?" Older adult 2:"Then I will have to use it."

Older adult 3: "If it [the use of an exoskeleton device] would be necessary, yes of course!"

[Would not use exoskeleton] One of the other participants (1/7) felt too old to try a new device, as she would not be going out anyway and the last (1/7) preferred doing everything herself instead of being dependent on exoskeleton technology. 
Older adult 5: "If it is somehow possible I would like to do things by myself as much as possible."

[Other older adults would use an exoskeleton] Some participants (3/7) argued that other older adults would be open to try an exoskeleton.

Older adult 2: "I think that they [older adults] are up for it [trying out an exoskeleton device]. ... Something new works wonders."

[Other older adults would not use an exoskeleton] In contrast, other participants could not think of anyone who would be needing such a device (3/7) or doubted that others would be willing to try it out (1/7).

Older adult 6: "I know some [older adults] that would be negative about it [exoskeletons]. They wouldn't feel like starting something anymore, you know?"

[Use of exoskeletons is person-dependent] All clinicians (6/6) indicated that the suitability of an exoskeleton device would be person-dependent.

Clinician 5: "I think that this will depend on the patient. Some people are open to everything that can help to make their life better, and others are fine with the way that things are. But I think that there is a large group of people that would be open to this [exoskeleton technology]."

[Acceptance of the need for assistive technology] Accepting the need for assistive technology was described to be a difficult process for both the older adult and their relatives, as this can be perceived as a sign of deteriorating health $(2 / 6)$.

Clinician 2: "[...] the biggest problem with rollators is that they [older adults] don't want to be seen with a rollator because it would make you an 'old person."”

This was illustrated by some older adult participants (2/7) mentioning that they would have to be "far gone" before needing an exoskeleton.

Older adult 4: "[ ...] I think it's nice that it's [exoskeletons] there but hopefully I don't have to use it. Yes, I need some things [assistive devices] but something like that [exoskeleton device], by then you are far gone, right?"

[Resistance to change] The clinicians experienced that change, especially involving new technology, was often met with resistance, which might cause people to refrain from adopting a mobility device (5/6).

Clinician 2: "In my experience, if we provide them [older adults] with things, those are of course not like these [exoskeleton devices], but just new things that can make life easier, then it is always a 'no' at first. Sometimes it's a process and eventually it becomes a 'yes' but sometimes the answer remains 'no.'"

[Exoskeletons are more suitable for younger generations] For this reason, exoskeletons were perceived to be more suitable for younger generations who are more familiar with the current state of technology (4/6), especially compared to people aged $80+(3 / 6)$.

Clinician 6: "I think that it [exoskeleton] is an unfamiliar device for this target group [older adults] and this target group already has problems with change. I would rather envision this [exoskeleton technology] to be suitable for someone who has been in a car accident and still has his whole life ahead of him." 
4.2.2 Design Requirements of Exoskeletons for Older Adults. [Inconspicuous design] Regarding the appearance of exoskeletons, older adult participants stated that the device should not be conspicuous (3/7). One participant (1/7) mentioned that she would try to conceal the device with her clothes as much as possible.

Older adult 6: "I wouldn't want the device to be too conspicuous. I would try to conceal it [exoskeleton] if I would use it."

The clinicians also emphasized the importance of an inconspicuous appearance (6/6). According to some of the clinicians, an exoskeleton that could be worn underneath regular clothing would be preferred by their clients, as this would make the device invisible to others (3/6). Alternatively, the exoskeleton could be worn as clothing, making the device less noticeable and look less complicated $(2 / 6)$.

Clinician 4: "[...] integration in clothing and so on or possibly something that could go underneath clothes or something like that. Yes, that would be a positive thing."

Moreover, the use of neutral colors (2/6) and a compact design (3/6) could help to make the device stand out less.

Clinician 6: "[ ...] currently it's [exoskeletons] very conspicuous and I think that if it would be less conspicuous ..., but also if it would be used more often, then it would also be less conspicuous, then it will become normal." Interviewer: "How could it be less conspicuous?" Clinician 6: "I think definitely more neutral colors and less ..., just the materials should be more compact."

[Utility over appearance] However, other older adults (3/7) indicated that they would wear an exoskeleton device if they would need it, regardless of its appearance.

Older adult 1: "I would wear it [exoskeleton], even if I would go to the market place."

[Ease of use] Other design requirements include that the device should be lightweight (2/6) and is easy to put on and take off, preferably independently (5/6).

Clinician 3: "I don't know how easy it is to connect or to put on [an exoskeleton]. That's of course a very relevant point in the context of older adults, to what degree they are still able to learn $[\ldots] "$

[Practical requirements] Practical issues raised by the clinicians included the costs involved for acquiring an exoskeleton (4/6) and the need for sufficient battery life; for example, to be able to use the device during shopping (2/6).

Clinician 1: "[...] but of course it is always the question: 'who will pay for it [exoskeleton]?" "

Clinician 5: "If you would be able to walk with it [exoskeleton] for a longer period of time, more people would use it when they want to walk longer distances, to the store or something. If you would walk for about 15 minutes and the thing would be empty, then it's no use."

4.2.3 Usages of Exoskeletons by Older Adults. [Using exoskeleton the whole day] Older adult participants indicated that they would be willing to wear an exoskeleton device during the entire day if necessary $(3 / 7)$. 
Older adult 1: "If someone's physical health allows that person to do things during the entire day, then there needs to be something [exoskeleton] that is comfortable and light enough to wear the whole day."

[Using exoskeleton for walking] Both the older adults (2/7) and the clinicians (5/6) indicated that the exoskeleton device would be used for support during walking. Two of the clinicians stated that an exoskeleton would be especially suitable to provide support when going outside.

Older adult 6: "[ ... ] if I can't walk anymore, let them give me something [exoskeleton] that allows me to walk a bit again. Don't lose hope."

Clinician 5: "I think when someone leaves the house to walk a longer distance. [...] Maybe they [older adults] will use it [exoskeleton] in their home but I think that they would use it more often to go outside, for longer distances."

[Using exoskeleton for non-walking actions] According to the clinicians, exoskeletons might also support older adults during lifting (1/6), prolonged standing (2/6), and climbing stairs (1/6).

Clinician 6: "[...] it is harder to perform activities from a wheelchair compared to a standing position."

[Using exoskeleton to increase endurance] The clinicians mentioned that an exoskeleton could help older adults to increase their endurance (4/6) and might be used during the recovery from injuries or illnesses $(2 / 6)$.

Clinician 6: "[ ...] I expect that people would be able to walk for longer periods of time and that walking will also be easier."

Clinician 3: "[...] in the recovery phase, for example for people who have been inactive for a prolonged period of time due to illness and who want to be active again. In this way they [older adults] can use it [exoskeleton] which can have a positive effect."

[Using exoskeleton to increase independence] Also, it was stated that exoskeleton technology could help older adults stay independent for longer $(2 / 6)$.

Clinician 4: "[...] people with reduced mobility or cardiovascular diseases or something like that will be able to live independently for a long time and will be able to take care of themselves this way [with the use of an exoskeleton]."

\section{DISCUSSION}

\subsection{Summary of Results}

Participants in this study were generally positive about the concept of exoskeleton technology. However, the older adults indicated that they would not currently need an exoskeleton device. The results indicate that whether the older adults would be willing to use an exoskeleton device is dependent on personal needs and preferences. If needed, most of the older adults would be willing to use an exoskeleton device, and some thought that their peers would use it as well. Others indicated to be reluctant to try the new technology and thought that other older adults would have no need for an exoskeleton device. Design requirements for exoskeletons focused mainly on two aspects: ease of use especially regarding (independent) donning and doffing and various ways to make the appearance of the device as inconspicuous as possible (e.g., compactness, neutral colors, and integration in clothing). Participants expected that an exoskeleton device would be used to 
support older adults during their daily activities, especially for walking (outside) but also during prolonged standing, lifting, and stair climbing.

\subsection{Acceptance of Exoskeleton Technology for Older Adults}

Older adults reported that they did not perceive the need for wearing an exoskeleton device despite their reported difficulties performing daily activities and their dependence on "traditional" mobility aids (see Figure 2). This paradoxical finding might be understood in light of previous work on Technology Acceptance Models (TAMs) that try to explain the complex relationship between the expectation of a person towards a technology (e.g., perceived usefulness and perceived ease of use) and their actual use [32]. It seems that older adults assess their health based on their current capabilities and assistive needs. As a result, the need for each new or additional assistive device might be perceived as a sign of deteriorating health. This is also reflected in remarks of the clinicians in this study: they mentioned that usually when they suggest a new assistive device, the first answer is "no." It is important for developers of mobility aids and clinicians who prescribe these devices to be sensitive to the stigmas that people might associate with the use of assistive devices [9]. Older adults and their relatives will most likely need time to accept the need for a (new) mobility aid and to get acquainted with the device [5]. A study on the perception of walkers among older adults showed that concerns about the need for a walker often subsided with time [24]. In contrast to our findings, the study (i.e., Reference [24]) also indicated that there is usually less resistance towards acquiring a second mobility device (e.g., getting a walker after having used a cane). This difference might be (partially) due to the fact that the older adult participants in this study were unfamiliar with exoskeleton technology, whereas walkers are commonly used among this user group. Nevertheless, the introduction of the electric bicycle has illustrated that older adults can be early adopters of new technology and that public opinion can change over time-from a mobility aid for people with disabilities to a bike that helps people of all ages to cover longer distances with ease [23].

Designers of exoskeletons can help to reduce the resistance towards wearing such a device by aiming for a discreet appearance. Providing users with customization options such as different colors could also help to make the device more acceptable [24]. Based on the results of this study, an exoskeleton with neutral colors would be preferred by older adults that would like the device to blend in with their regular clothing. Additionally, other colors might be offered to serve users that would like to wear an exoskeleton in their favorite color. Moreover, co-design methods can be used to involve older adults and other stakeholders in the development process (e.g., see Reference [6]).

Ease of use has also been identified in previous studies [25, 38] as an important design requirement for exoskeleton devices that are meant to be used in daily living. Older adults might encounter problems putting on and taking off an exoskeleton device due to joint stiffness. Consequently, designers of exoskeletons for older adults should take age-related loss of range-of-motion into account.

From an engineering perspective, there are still technical challenges to overcome with regards to power supply, lightweight actuators, and efficient transmissions [8]. Moreover, soft exoskeletons will also require flexible sensing and actuation techniques [25, 36]. With the current state of technology, a hip exoskeleton (e.g., see Figure 1, right) might be the best trade-off for older adult users considering their preferences regarding the appearance of the device (i.e., compact and as inconspicuous as possible) compared to the available sizable rigid lower-limb exoskeletons (e.g., see Figure 1, left) and experimental exosuits (e.g., Reference [2]). It should be noted, however, that most currently available exoskeletons are unable to support gait stability [38]. Therefore, older adults with gait instability will still require the use of another mobility aid for stability, such as 
a rollator. An exception is the REX powered lower-limb exoskeleton by Rex Bionics, ${ }^{5}$ which is self-supporting, requiring no use of crutches or walker, albeit at the cost of a more bulky design.

\subsection{Integration of Exoskeleton Technology into the Lives of Older Adults}

The findings of this study indicated that the suitability of an exoskeleton device will be persondependent. Indeed, previous studies emphasize the importance of matching the assistive device to the capabilities of the user, both on a physical and cognitive level, and their environment $[5,25$, 34]. For this reason, it is important to actively include older adults in the decision about acquiring an assistive device [24]. The match between user and technology can be optimized by trying to understand what the assistive device means to the user, which purpose it is expected to serve, and how the device can be incorporated into the daily life and activities of a person [34]. For example, viewing a mobility aid as a symbol of independence that enables its user to perform daily activities would ease the acceptance of a device [24]. The older adults in this study seemed to attribute meaning to their mobility aids, such as the feeling of safety they get from using the device or the freedom to go outside and cover longer distances but also a feeling of dependency, which complicates their lives, as they have to take the device with them wherever they go. Previous studies showed that older adults might differ in the value that they place on their level of autonomy and the importance of the appearance of a mobility aid [24, 25]. Some older adults in this study also clearly valued the ability to be mobile and perform daily activities over concerns about how they would look wearing an exoskeleton device. To further help older adults to integrate a mobility device into their daily life, new users should be provided with clear instructions, contact information in case of problems, help to set up the device, and training on how to use it [5]. In addition, followup visits for answering questions and solving problems cannot only improve the effectiveness of the device but also positively influence user satisfaction [5].

One of the technical aspects that currently limits use in daily life is the immaturity of the algorithms that control exoskeleton devices to perform different actions at varying speeds and adapt to different terrain [7]. Moreover, exoskeletons are being developed to either enhance human performance or to compensate for loss in physical functioning [8]. However, if exoskeleton technology becomes more readily available to consumers in the near future, users might be inclined to also use such a device to support them in tasks that they would (still) be able to perform independently. To avoid unnecessary disuse of body function, algorithms are being developed for the control of exoskeletons to provide the appropriate level of support that is "assistance as needed" [1]. As this method for determining the need for assistance will most likely not prevent deliberate "misuse," developers of exoskeleton technology should be mindful of possible adverse effects of the use of such devices. Furthermore, it should be noted that current exoskeleton devices require supervised use, which limits the feasibility of use outside clinical settings [7].

\subsection{Considerations Regarding the Study}

It is important to note that participation in the study was on a voluntary basis. As a result, the participants might have been more open towards new technologies. Data was collected based on availability of participants instead of until saturation was reached. Further research will be required to verify the transferability of the findings. In this study, we have used triangulation by interviewing two different groups of stakeholders. However, the use of multiple trustworthiness strategies such as asking study participants to provide feedback on transcripts of the interview to verify whether their intentions were properly captured would have enhanced the quality of the data. In this study, participants were asked to express their views on exoskeleton technology based

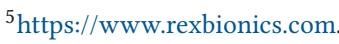


on an explanation supported with pictures and video of exoskeleton devices. As a result, it might have been difficult for participants to get a complete understanding of the advantages and disadvantages of the use of exoskeletons. With the advances in exoskeleton technology, it will likely become feasible in the near future to study the use of these devices in a home environment. Longterm studies will be necessary to get a better understanding of how exoskeleton technology can support older adults in their daily activities and whether their use will increase physical activity among users.

\section{CONCLUSION}

Reactions towards exoskeleton technology were generally positive, indicating that exoskeletons can be a valuable addition to existing mobility devices to assist older adults with daily living. However, the older adults in this study did not perceive the current need for using an exoskeleton device. Moreover, the results show the willingness to use exoskeleton technology to support older adults in their daily activities if needed will vary from person to person.

To successfully integrate exoskeleton technology into the lives of older adults, there are still a lot of challenges to overcome from the engineering perspective as well as the user perspective. Developers of exoskeletons will have to design a device that is easy to don and doff that can preferably also be used independently by frail older adults. In addition to an intuitive design, training should be offered to ensure that the exoskeleton device is properly used and has the desired effect. The need for assistive technology might be perceived as a sign of deteriorating health, forming a barrier to its adoption. Therefore, older adults should be actively involved in the decision to acquire a mobility aid and should receive expert advice to make an informed decision. Moreover, giving the opportunity to try out a device will help to determine if the mobility aid suits the needs of the user. Developers of exoskeletons can ease acceptance by designing a device that blends in with the regular clothing of the user or can be worn underneath. In the end, it will be dependent onpersonal preference, health condition, and living situation of a person whether the use of an exoskeleton device is a suitable option. Offering older adults a diverse range of assistive devices can help to suit their individual needs.

\section{ACKNOWLEDGMENTS}

The authors would like to express their gratitude to all participants for their commitment to the study and to the staff of De Posten and Alifa for their cooperation.

\section{REFERENCES}

[1] Maarten Afschrift, Friedl De Groote, Joris De Schutter, and Ilse Jonkers. 2014. The effect of muscle weakness on the capability gap during gross motor function: A simulation study supporting design criteria for exoskeletons of the lower limb. Biomed. Eng. Online 13, Article 111 (2014). DOI : https://doi.org/10.1186/1475-925X-13-111

[2] Alan T. Asbeck, Stefano M. M. De Rossi, Ignacio Galiana, Ye Ding, and Conor J. Walsh. 2014. Stronger, smarter, softer: Next-generation wearable robots. IEEE Robot. Auto. Mag. 21, 4 (2014), 22-33. DOI : https://doi.org/10.1109/MRA.2014. 2360283

[3] Johannes W. J. Bijlsma, C. H. B. Oude Heuvel, and Ap Zaalberg. 1990. Development and validation of the Dutch questionnaire capacities of daily life (VDF) for patients with rheumatoid arthritis. J. Rehab. Sci. 3 (1990), 71-74.

[4] Jaimie F. Borisoff, Johanne Mattie, and Vince Rafer. 2013. Concept proposal for a detachable exoskeleton-wheelchair to improve mobility and health. In Proceedings of the IEEE International Conference on Rehabilitation Robotics (ICORR'13). IEEE, 1-6. DOI : https://doi.org/10.1109/ICORR.2013.6650396

[5] Åse Brandt, Susanne Iwarsson, and Agneta Ståhl. 2003. Satisfaction with rollators among community-living users: A follow-up study. Disab. Rehab. 25, 7 (2003), 343-353. DOI : https://doi.org/10.1080/0963828021000058495

[6] Peta Bush and Simone ten Hompel. 2017. An integrated craft and design approach for wearable orthoses. Design Health 1, 1 (2017), 86-104. DOI : https://doi.org/10.1080/24735132.2017.1299439 
[7] Rachel E. Cowan, Benjamin J. Fregly, Michael L. Boninger, Leighton Chan, Mary M. Rodgers, and David J. Reinkensmeyer. 2012. Recent trends in assistive technology for mobility. F. Neuroeng. Rehab. 9, 1, Article 20 (2012). DOI : https://doi.org/10.1186/1743-0003-9-20

[8] Aaron M. Dollar and Hugh Herr. 2008. Lower extremity exoskeletons and active orthoses: Challenges and state-ofthe-art. IEEE Trans. Robot. 24, 1 (2008), 144-158. DOI : https://doi.org/10.1109/TRO.2008.915453

[9] Deborah Hill, Catherine S. Holloway, Dafne Z. Morgado Ramirez, Peter Smitham, and Yannis Pappas. 2017. What are user perspectives of exoskeleton technology? A literature review. Int. F. Technol. Assess. Health Care 33, 2 (2017), 160-167. DOI : https://doi.org/10.1017/S0266462317000460

[10] Honda. 2013. Honda's Stride Management Assist. Retrieved from https://www.autoblog.com/2013/11/17/hondawalking-assist-device-testing-at-rehabilitation-institute.

[11] Shanhai Jin, Noriyasu Iwamoto, Kazunobu Hashimoto, and Motoji Yamamoto. 2017. Experimental evaluation of energy efficiency for a soft wearable robotic suit. IEEE Trans. Neural Syst. Rehab. Eng. 25, 8 (2017), 1192-1201. DOI : https://doi.org/10.1109/TNSRE.2016.2613886

[12] Kyoungchul Kong and Doyoung Jeon. 2006. Design and control of an exoskeleton for the elderly and patients. IEEE/ASME Trans. Mechatron. 11, 4 (2006), 428-432. DOI : https://doi.org/10.1109/TMECH.2006.878550

[13] Hwang-Jae Lee, Suhyun Lee, Won Hyuk Chang, Keehong Seo, Youngbo Shim, Byung-Ok Choi, et al. 2017. A wearable hip assist robot can improve gait function and cardiopulmonary metabolic efficiency in elderly adults. IEEE Trans. Neural Syst. Rehab. Eng. 25, 9 (2017), 1549-1557. DOI : https://doi.org/10.1109/TNSRE.2017.2664801

[14] Su-Hyun Lee, Hwang-Jae Lee, Won Hyuk Chang, Byung-Ok Choi, Jusuk Lee, Jeonghun Kim, et al. 2017. Gait performance and foot pressure distribution during wearable robot-assisted gait in elderly adults. f. Neuroeng. Rehab. 14, 123 (2017). DOI : https://doi.org/10.1186/s12984-017-0333-z

[15] Maria M. Martins, Cristina P. Santos, Anselmo Frizera-Neto, and Ramón Ceres. 2012. Assistive mobility devices focusing on smart walkers: Classification and review. Robot. Autonom. Syst. 60, 4 (2012), 548-562. DOI: https:// doi.org/10.1016/j.robot.2011.11.015

[16] Medipoint. 2018. Image cane. Retrieved from https://www.medipoint.nl/producten/wandelstok-caremart-tadvance. html.

[17] Medipoint. [n. d.]. Image crutch. Retrieved from https://www.medipoint.nl/producten/elleboogkruk-anatomischdubbel-verstelbaar.html.

[18] Medipoint. [n. d.]. Image mobility scooter. Retrieved from https://www.medipoint.nl/producten/scootmobiel-colibri. html.

[19] Medipoint. [n. d.]. Image rollator. Retrieved from https://www.medipoint.nl/producten/rollator-basic-1.html.

[20] Medipoint. [n. d.]. Image walking frame. Retrieved from https://www.medipoint.nl/producten/looprek-travel-walker. html.

[21] Medipoint. [n. d.]. Image wheelchair. Retrieved from https://www.medipoint.nl/producten/rolstoel-multimotion-m9. html.

[22] Leonard O’Sullivan, Valerie Power, Gurvinder Virk, Nauman Masud, Usman Haider, Simon Christensen, et al. 2015. End user needs elicitation for a full-body exoskeleton to assist the elderly. Procedia Manufact. 3 (2015), 1403-1409. DOI : https://doi.org/10.1016/j.promfg.2015.07.302

[23] Alexander Peine, Vivette van Cooten, and Louis Neven. 2017. Rejuvenating design: Bikes, batteries, and older adopters in the diffusion of e-bikes. Sci., Technol., Human Values 42, 3 (2017), 429-459. DOI : https://doi.org/10.1177/ 0162243916664589

[24] Ken Pippin and Geoffrey R. Femie. 1997. Designing devices that are acceptable to the frail elderly: A new understanding based upon how older people perceive a walker. Technol. Disab. 7, 1-2 (1997), 93-102. DOI: https:// doi.org/10.3233/TAD-1997-71-211

[25] Valerie Power, Leonard O’Sullivan, Adam de Eyto, Samuel Schülein, Corien Nikamp, Christoph Bauer, et al. 2016. Exploring user requirements for a lower body soft exoskeleton to assist mobility. In Proceedings of the 9th ACM International Conference on PErvasive Technologies Related to Assistive Environments (PETRA'16). ACM, Article 69. DOI : https://doi.org/10.1145/2910674.2935827

[26] R Core Team. 2017. R: A Language and Environment for Statistical Computing. R Foundation for Statistical Computing, Vienna, Austria. Retrieved from https://www.R-project.org/.

[27] ReWalk. 2014. ReWalk ${ }^{\mathrm{TM}}$ exoskeleton. Retrieved from https://www.medgadget.com/2014/06/rewalk-exoskeletonsystem-cleared-by-fda-for-home-use.html.

[28] Erik Scherder, Rogier Scherder, Lot Verburgh, Marsh Königs, Marco Blom, Arthur F. Kramer, and Laura Eggermont. 2014. Executive functions of sedentary elderly may benefit from walking: A systematic review and meta-analysis. Amer. F. Ger. Psych. 22, 8 (2014), 782-791. DOI : https://doi.org/10.1016/j.jagp.2012.12.026

[29] Kai Schmidt, Jaime E. Duarte, Martin Grimmer, Alejandro Sancho-Puchades, Haiqi Wei, Chris S. Easthope, et al. 2017. The Myosuit: Bi-articular anti-gravity exosuit that reduces hip extensor activity in sitting transfers. Front. Neurorobot. 11, Article 57 (2017). DOI : https://doi.org/10.3389/fnbot.2017.00057 
[30] Hiroyuki Shimada, Takashi Hirata, Yuichi Kimura, Takako Naka, Keishiro Kikuchi, Keiichi Oda, et al. 2009. Effects of a robotic walking exercise on walking performance in community-dwelling elderly adults. Geriat. Gerontol. Int. 9, 4 (2009), 372-381. DOI : https://doi.org/10.1111/j.1447-0594.2009.00546.x

[31] Hiroyuki Shimada, Takao Suzuki, Yuichi Kimura, Takashi Hirata, Miho Sugiura, Yosuke Endo, et al. 2008. Effects of an automated stride assistance system on walking parameters and muscular glucose metabolism in elderly adults. Brit. J. Sports Med. 42, 11 (2008), 922-929. DOI : https://doi.org/10.1136/bjsm.2007.039453

[32] Linda Shore, Valerie Power, Adam de Eyto, and Leonard W. O’Sullivan. 2018. Technology acceptance and user-centred design of assistive exoskeletons for older adults: A commentary. Robotics 7, 1, Article 3 (2018). DOI: https://doi.org/ 10.3390/robotics7010003

[33] Maria A. F. Singh. 2002. Exercise comes of age: Rationale and recommendations for a geriatric exercise prescription. f. Gerontol.: Series A 57, 5 (2002), M262-M282. DOI : https://doi.org/10.1093/gerona/57.5.M262

[34] Bronwen Thomas, Denise Connelly, and Debbie Laliberte-Rudman. 2008. The impact and use of walkers among older adults: A pilot. Phys. Occupat. Ther. Geriat. 27, 1 (2008), 36-72. DOI : https://doi.org/10.1080/02703180802275327

[35] David R. Thomas. 2006. A general inductive approach for analyzing qualitative evaluation data. Amer. 7. Eval. 27, 2 (2006), 237-246.

[36] Allan J. Veale and Shane Q. Xie. 2016. Towards compliant and wearable robotic orthoses: A review of current and emerging actuator technologies. Med. Eng. Phys. 38, 4 (2016), 317-325. DOI : https://doi.org/10.1016/j.medengphy.2016. 01.010

[37] Sandra C. Webber, Michelle M. Porter, and Verena H. Menec. 2010. Mobility in older adults: A comprehensive framework. The Gerontologist 50, 4 (2010), 443-450. DOI : https://doi.org/10.1093/geront/gnq013

[38] Jamie Wolff, Claire Parker, Jaimie Borisoff, W. Ben Mortenson, and Johanne Mattie. 2014. A survey of stakeholder perspectives on exoskeleton technology. F. Neuroeng. Rehab. 11, 169 (2014). DOI:https://doi.org/10.1186/ 1743-0003-11-169

[39] World Health Organization. 2015. World Report on Ageing and Health.World Health Organization. Retrieved from https:/www.who.int/ageing/events/world-report-2015-launch/en/.

[40] Hye A. Yeom, Julie Fleury, and Colleen Keller. 2008. Risk factors for mobility limitation in community-dwelling older adults: A social ecological perspective. Geriat. Nurs. 29, 2 (2008), 133-140. DOI: https://doi.org/10.1016/j.gerinurse. 2007.07.002

Received April 2018; revised November 2018; accepted January 2019 